\title{
Historische Epistemologie
}

Edgar Forster \& Tanja Obex

In seinem Buch „Was heißt ,soziale Konstruktion“?“ untersucht Hacking (1999) die „Konjunktur einer Kampfvokabel in den Wissenschaften“ und fragt, ob Geisteskrankheiten biologisch oder konstruiert sind. Ebenfalls um die Jahrtausendwende veröffentlicht Rheinberger (2006) eine Studie über „Die Evolution des Genbegriffs“. Bereits siebzig Jahre früher hat Fleck (1935) rekonstruiert, wie der Syphilisbegriff entstand. Und Daston und Galison (2007) zeigen in einer historischen Analyse, wie der Begriff Objektivität um 1860 der Naturwahrheit den Platz als Wissenschaftsideal streitig gemacht hat. Alle vier Studien repräsentieren eine Forschungstradition an der Schnittstelle von Wissenschaftsforschung und -philosophie, die unter dem Namen „historische Epistemologie“ bekannt geworden ist und im deutschsprachigen Raum seit etwa dreißig Jahren eine Konjunktur ${ }^{1}$ erlebt.

Lecourt (1969) hat den Begriff „historische Epistemologie“ im Titel seines Buches „L’épistémologie historique de Gaston Bachelard“ verwendet, um Bachelards Denken zu charakterisieren. ${ }^{2}$ Gegen die damalige Tradition und das wissenschaftliche Fortschrittsdenken sah es Bachelard als Aufgabe der Erkenntnistheorie, die historischen Bedingungen der Produktion wissenschaftlicher Erkenntnisse und hier vor allem Brüche und Neuorganisationen in der Wissenschaftsentwicklung zu untersuchen. Bei allen Unterschieden teilen Bachelard, Canguilhem und Foucault, die zu den prominentesten Vertretern der französischen Wissenschaftsgeschichte und den Begründern der historischen Epistemologie zählen, die Annahme, dass Wissen immer in spezifischen sozialen und historischen, aber auch wissenschaftlichen und technischen Kontexten entsteht.

In diesem Beitrag rekonstruieren wir historische Epistemologie nicht nur als eine spezifisch französische Variante der Wissenschaftsgeschichte und -philosophie, sondern wir folgen Rheinberger (2017, S. 33f.), der historische Epistemologie als ein transdisziplinäres und internationales Phänomen von wissenschaftlichen und gesellschaftlichen Entwicklungen begreift, in denen Wissen und ihre Produktionsformen, der Kampf um ,richtiges‘ Wissen und Wissenspolitik eine hohe gesellschaftliche Bedeutung erfahren haben.

\footnotetext{
${ }^{1}$ Das findet Niederschlag in akademischen Institutionen wie dem Max-Planck-Institut für Wissenschaftsgeschichte in Berlin, in Handbüchern (Sommer et al. 2017) und Beiträgen zu solchen (Rheinberger 2017; Wulz 2014) sowie in einführenden Darstellungen (Rheinberger 2007).

${ }^{2}$ Canguilhem (1979, S. 7-21) hat bereits 1963 in einem Text über „L’histoire des sciences dans l’œuvre épistémologique de Gaston Bachelard“ den Begriff „historische Epistemologie“ verwendet (zur Geschichte des Begriffs vgl. Schmidgen et al. 2012, S. 20; zur Kritik der beliebigen Verwendung des Begriffs vgl. Gingras 2010).
} 
Im Feld der Analyse von Wissensproduktion und der Verschränkung von Wissenschaft, Technik und Gesellschaft entwickelt sich die historische Epistemologie in der Auseinandersetzung mit verwandten, oft nicht scharf voneinander trennenden Forschungsprogrammen weiter. Dazu zählen neben Philosophie und Geschichte vor allem Wissenschaftssoziologie, Wissensgeschichte (Renn 2012; Sarasin 2011) sowie Science and Technologie Studies (Bauer et al. 2017; Daston 2009; Dear und Jasanoff 2010). An der Frage nach der Bedeutung des Politischen in wissenschaftshistorischen Analysen werden solche Überschneidungen deutlich: Speich Chassé und Gugerli (2012) grenzen Wissensgeschichte vom Feld der Wissenschaftsgeschichte ab. Diese reflektiere zwar „den gesellschaftlichen Resonanzraum der Erkenntnisproduktion“ (ebd., S. 93), mache aber politische und wirtschaftliche Machtverhältnisse nicht zum Gegenstand ihrer Forschung. Demgegenüber befasst sich Roelcke (2010) aus der Perspektive der Medizin mit historischer Epistemologie und plädiert dafür, „Politik als konstitutiven Bestandteil von wissenschaftlicher Praxis in all ihren Stadien zu begreifen“ (ebd., S. 187). Das Politische sei keine zusätzliche Analysedimension, sondern jedem wissenschaftlichen Handeln immanent.

Für eine präzisere Bestimmung der historischen Epistemologie werden wir im Folgenden an die Ideen der Vertreter der französischen Tradition der historischen Epistemologie und an den polnischen Bakteriologen Fleck erinnern, im Anschluss daran aktuelle Diskussionen über die Analyse von Wissensproduktion darstellen und schließlich danach fragen, wie die historische Epistemologie in der deutschsprachigen Erziehungswissenschaft aufgenommen wird.

\section{Die Formierung der historischen Epistemologie}

Die Werke von Bachelard, Canguilhem, Foucault und Fleck gelten als wegweisend für die Formierung der historischen Epistemologie. Wir beschränken uns hier darauf, einige ihrer zentralen Themen darzustellen.

(a) Epistemische Brüche und Realisierungen von wissenschaftlichen Objekten (Bachelard): Eine Philosophie der Wissenschaften müsse zeigen, unter welchen subjektiven und objektiven Bedingungen allgemeine Prinzipien zu partikulären Ergebnissen und umgekehrt partikuläre Ergebnisse zu Verallgemeinerungen Anlass geben. Mit diesem Programm grenzt Gaston Bachelard (1884-1962) in Die Philosophie des Nein (1980/1940) seine Wissenschaftsphilosophie von der damals vorherrschenden Philosophie der Wissenschaften ab, die sich auf die Untersuchung allgemeiner Erkenntnisprinzipien durch die Philosophie und auf die Untersuchung partikulärer Ergebnisse durch die Wissenschaft beschränkt hat. 
Die Bildung des wissenschaftlichen Geistes geht mit einem epistemologischen Bruch einher. Unmittelbaren Erfahrungen, ersten Systembildungen und wissenschaftlichen Systemen sei zu misstrauen; sie enthalten einen „Trägheitsfaktor“, der die Reflexion beschränke (Bachelard 1987, S. 54). Was der Mensch in seiner wissenschaftlichen Technik mache, sei in der Natur nicht einfach vorfindbar, sondern müsse durch Phänomenotechniken - das sind materielle Instrumente und Experimentaltechniken - hergestellt werden. Rheinberger (2007) hebt Bachelards Begriff der „Realisierung“ als einen Schlüsselbegriff für das Konzept der historischen Epistemologie hervor: „Es geht also weniger um die Wirklichkeit und ihre Erkenntnis um das, was ist - als vielmehr um einen Prozess der ,Verwirklichung', um das, was sein kann“ (ebd., S. 39).

Bachelards Denken ist durch Aufklärung und Fortschritt geprägt. Er unterscheidet streng zwischen wissenschaftlichem Wissen und Alltagswissen und hat die historische Epistemologie für hochformalisierte Wissenschaften wie die Physik entwickelt. Canguilhem und Foucault erweitern das Feld auf die Wissenschaft vom Leben und Geschichte. Hier spielen Begriffe gegenüber nur schwach formalisierten Theorien eine größere Rolle (Foucault 2003; Müller und Schmieder 2016, Kap. IV). Das entspricht Bachelards (1980, S. 27) Forderung, dass jeder Begriff, jede Hypothese und jedes Problem seine eigene historische Epistemologie benötige.

(b) Das Objekt der Wissensgeschichte (Canguilhem): Wie ein ,natürliches“ oder ,gegebenes“ Objekt zum Gegenstand der Wissenschaft gemacht wird, ist die Forschungsperspektive der Wissenschaftsgeschichte von Georges Canguilhem (1904-1995). Während ein wissenschaftliches Objekt über die Theorie mit dem natürlichen Gegenstand verbunden ist, bezieht sich das Objekt der Wissenschaftsgeschichte auf den sekundären, nicht natürlichen, kulturellen Gegenstand, aber leitet sich ebenso wenig von diesem ab wie der wissenschaftliche Gegenstand vom natürlichen Gegenstand. „Der Gegenstand der Wissenschaftsgeschichte ist in der Tat die Geschichtlichkeit des wissenschaftlichen Diskurses“ (Canguilhem 1979, S. 30). Zwischen den verschiedenen Objekten besteht keine Ableitungsbeziehung, und Wissenschaftsgeschichte konstituiert den Bereich, „in den die von der wissenschaftlichen Praxis und ihrer Entwicklung aufgeworfenen theoretischen Fragen hineingehören“ (ebd., S. 31). Der Gegenstand kann nur dadurch bestimmt werden, dass Entscheidungen getroffen werden, die dem Gegenstand „sein Interesse und seine Bedeutung“ (ebd., S. 30f.) zuweisen. Der Diskurs produziert mit dem Gegenstand auch seine Interessen und Bedeutungen (Borck et al. 2005, S. 15; Foucault 1981, S. 262-265; Roelcke 2010).

Die deutschsprachige Canguilhem-Rezeption begann zwar schon Mitte der 1970er Jahre mit der Übersetzung von „Le normal et le pathologique“ (Canguilhem 1974), aber für die histori- 
sche Epistemologie ist vor allem die Publikation einer Auswahl von Aufsätzen unter dem Titel „Wissenschaftsgeschichte und Epistemologie“ (Canguilhem 1979) bedeutsam (vgl. Limoges 2012). Größere Aufmerksamkeit hat Canguilhem zum einen durch Wissenschaftsdebatten im Anschluss an Kuhns Werk „Die Struktur wissenschaftlicher Revolutionen“ (1973) und zum anderen durch Foucault erfahren. Nach der Jahrtausendwende war die Rezeption eng mit dem Aufstieg der Historischen Epistemologie und den Science and Technology Studies verbunden, die an Canguilhems Interesse für Fragen der Technik anknüpfen (Schmidgen et al. 2012; Rheinberger 2007, 2006; Borck et al. 2005).

(c) Archäologie des Wissens (Foucault): Michel Foucault (1926-1984) ist längst ein Klassiker, dessen Werk zwar starke Bezüge zur Wissenschaftsgeschichte und zu epistemologischen Fragen aufweist, aber diese spielen in der erziehungswissenschaftlichen Rezeption gegenüber den Konzepten von Macht, Subjektivierung oder Gouvernementalität nur eine untergeordnete Rolle (vgl. z.B. Messerschmidt 2012; Ricken und Rieger-Ladich 2004). Wir müssen uns hier darauf beschränken, den Begriff „Episteme“ in der „Archäologie des Wissens“ (Foucault 1981) darzustellen, in der Foucault eine Dreiteilung von Praxis, Wissen und Wissenschaft vornimmt. Jede Praxis ist diskursiv verfasst, indem sie Positivitäten, die noch keine Erkenntnisform bilden, nach bestimmten Regeln zu „Gegenstandsgruppen, Äußerungsmengen, Begriffsbündel und Serien theoretischer Wahlmöglichkeiten“ (ebd., S. 258) verknüpft. Diese konfigurierten Elemente nennt Foucault „Wissen“ (ebd., S. 259). Eine erste „Schwelle der Positivität“ (ebd., S. 265) gibt es dann, wenn sich aus der Fülle der Positivitäten eine diskursive Praxis herausbildet.

Nach Foucault lassen sich verschiedene Wissensfelder unterscheiden, aber es gibt keinen natürlichen Übergang von einem Feld zum nächsten, und nicht alle Wissensfelder bilden sich zur gleichen Zeit und auf die gleiche Weise heraus. Formal folgt auf die Schwelle der Positivität eine „Schwelle der Epistemologisierung“ (ebd., S. 266). Sie ist erreicht, wenn ein Diskurs im Hinblick auf ein Wissen eine regulierende Funktion ausübt, also etwa Kohärenznormen zur Geltung bringt. Und wenn ein solcher Diskurs bestimmten formalen Kriterien gehorcht, dann wäre die „Schwelle der Wissenschaftlichkeit“ (ebd.) überschritten. Schließlich lässt sich noch ein weiteres Wissensfeld identifizieren, das die Regeln der Wissenschaftlichkeit definiert (Deleuze 1987, S. 69-98).

Die verschiedenen Wissensfelder erlauben verschiedene Formen der historischen Analyse. Historische Epistemologie ist die Analyse der Episteme oder archäologische Geschichte. Als eine Form der Wissenschaftsgeschichte fragt sie, wie ein Diskurs die Normen der Wissenschaftlichkeit erreicht (Foucault 1981, S. 271f.), wie etwa ein pädagogischer Diskurs, der 
nicht nur ein Wissensdiskurs ist, sondern diesen auch reguliert, die Schwelle zur Wissenschaftlichkeit überschreitet. Episteme zu analysieren, heißt, die Gesamtheit der Beziehungen zu untersuchen, die man in einer gegebenen Zeit innerhalb der Wissenschaften entdecken kann; mit anderen Worten, die diskursiven Regelmäßigkeiten eines wissenschaftlichen Diskurses.

(d) Soziale, psychologische und historische Bedingtheit der Erkenntnis (Fleck): Ludwik Flecks (1896-1961) Werk „Entstehung und Entwicklung einer wissenschaftlichen Tatsache“ (Fleck 1980), in dem er die Entstehung und Stabilisierung von Erfahrungstatsachen am Beispiel der Beziehung zwischen der sogenannten Wassermann Reaktion und der Syphilis rekonstruiert, gilt als ein wesentlicher Beitrag zur historischen Epistemologie. Seine Erkenntnistheorie betont die sozialen, psychologischen und historischen Bedingungen der Wissensproduktion. Fleck stellt sich damit in seiner „vergleichenden Erkenntnistheorie“ (ebd., S. 34) gegen die Forderung der logischen Empiristen, den Entdeckungszusammenhang in wissenschaftlichen Untersuchungen auszuklammern (ebd., S. 31). Dieser Zugang ermögliche es, erkenntnistheoretisch einen „weniger egozentrischen, allgemeineren Standpunkt“ (ebd., S. 34) einzunehmen. Gegen die zeitgenössische Tendenz, das epistemologische Subjekt als „absolut, unveränderlich und allgemein“ (Fleck 2011, S. 260) zu setzen, führt Fleck neben Subjekt und Objekt das Wissen als drittes Glied im Erkenntnisprozess ein.

Wissenschaft ist für Fleck eine Tätigkeit von Forscherinnen- und Forschergemeinschaften, die Denkkollektive mit einem spezifischen Denkstil bilden (Fleck (1980, S. 54f.). Wissenschaftliches Wissen entstehe und verfestige sich im Rahmen historisch kontingenter Settings, von Denkkollektiven und Denkstilen, unter Berücksichtigung kultureller Wissensbestände, den sogenannten „Urideen“, die er als unklare vorwissenschaftliche Ideen, die Sachverhalte erklären, ohne sie legitimieren zu müssen, beschreibt. Flecks Denken richtet sich gegen den als selbstverständlich angenommenen positivistischen Tatsachenbegriff: Die Erkenntnistheorie „zieht fast ausschließlich uralte Tatsachen des Alltags oder der klassischen Physik als einzig sichere und der Untersuchung würdige in Betracht. So findet schon zu Beginn der Untersuchung eine naive Wertung statt, die sich durch Verflachung der Ergebnisse rächt“ (ebd., S. 1). Um die Unvoreingenommenheit im erkenntnistheoretischen Prozess und einen Forschungsfortschritt zu gewährleisten, schlägt Fleck vor, auf Tatsachen zurückzugreifen, „deren Entdeckung nicht weit zurückliegt und die noch nicht allseitig zu erkenntnistheoretischen Zwecken ausgenützt wurden“ (ebd.). 


\section{Wissen, Repräsentation, Ethik}

Welches Wissen ist Gegenstand der historischen Epistemologie? Was ist ihr wissenschaftliches Objekt? Und weshalb hat sie eine ethische Dimension? Diese drei Fragen bestimmen die gegenwärtige epistemologische Debatte in der Wissenschaftsforschung.

(a) Wissen: Bachelards Version der historischen Epistemologie beginnt mit dem Erkenntnisbruch zwischen Meinung und Wissen, aber Kritiker wie Latour (1995) weisen scharfe Trennungen zwischen Wissensformen, zwischen Internalismus und Externalismus oder zwischen Wissenschaft und sozialem oder politischem Umfeld als Erfindungen der Moderne zurück. Für ,weiche‘ Sozialwissenschaften wie die Erziehungswissenschaft, deren Theorien einen geringen Formalisierungsgrad aufweisen, sind theoretische Ansätze vielversprechender, die das Wissen als Teil eines gesamtgesellschaftlichen Prozesses begreifen. Ein offener Begriff des Wissens ohne vorgängige Abgrenzung von Information, Glauben, Erfahrung und ähnlichen Begriffen ermöglicht Erkenntnisse darüber, wie eine Gesellschaft den Begriff des Wissens hervorbringt und ausdifferenziert (Speich Chassé und Gugerli 2012, S. 95).

(b) Repräsentation: Existieren Mikroorganismen, die Milchsäure erzeugen, vor ihrer Entdeckung oder nicht? Das berührt Fragen nach der Realität vor und außerhalb eines Forschungsprozesses. Für Latour und Haraway sind bei allen Unterschieden Fragen nach dem Verhältnis zwischen Dingen und Zeichen unergiebig. Latour (1995, S. 71-77) spricht von „QuasiObjekten“, und Haraway (1995, S. 109) zufolge sind wissenschaftliche Objekte „Ablagerungen von Interaktionen und Beziehungen. Nichts existiert vor dieser Relationalität. [...] Subjekte und Objekte sind das Ergebnis diskursiver Konstruktionen.“

Der Übergang von einer epistemologischen zu einer ontologischen Betrachtung der Wissensproduktion bestimmt auch die Arbeiten von Barad. Sie ficht eine Konzeption von Realität an, die entweder als vorgängig oder als außerhalb von Sprache begriffen wird. Realität bestehe, so Barad (2015, S. 37), aus „Dingen-in-Phänomenen“. Dieser Realismus bezieht sich auf „die realen Konsequenzen, Interventionen, kreativen Möglichkeiten und Verantwortlichkeiten des Intraagierens in der Welt“ (ebd., S. 61f.). Daraus folgt die Notwendigkeit einer Ethik des Wissens, denn das konstruierte Wissen hat reale materielle Konsequenzen.

(c) Ethik: Canguilhem (1979, S. 40) hält das Konzept der „Vergangenheit einer Wissenschaft“ für ein Vulgärkonzept, wenn man es absolut nimmt. Es sei ein illusorischer Versuch, die Totalität des Vergangenen abbilden zu wollen. Wissenschaftshistorikerinnen und -historiker wählen vielmehr eine Darstellung, um einen ,Fortschritt‘ oder eine Verschiebung sichtbar zu machen, „dessen Ausdruck ja gerade den aktuellen Gegenstand des Interesses ausmacht“ (ebd.). Von der historischen Epistemologie, so Canguilhem, könne die Wissenschaftsgeschichte eine 
„Sittenlehre“ erwarten, „wenn man auf der imaginären Ebene der gesamten Vergangenheit derartige zurückschreitende Verschiebungen vornehmen will““ (ebd.). Auf ähnliche Weise thematisiert Haraway (1995) die ethische Dimension der Wissensproduktion. Wissensansprüche sind kontingent und deswegen seien Wissenschaftlerinnen und Wissenschaftler rechenschaftspflichtig und müssen „eine kritische Praxis zur Wahrnehmung unserer eigenen bedeutungserzeugenden, ,semiotischen Technologien` entwickeln“ (ebd., S. 78). Dieses wissenschaftliche Engagement müsse einem „nicht-sinnlosen Engagement für Darstellungen verpflichtet sein [...], die einer ,wirklichen` Welt die Treue halten“ (ebd.). Positionierung sei daher die entscheidende wissensbegründende Praktik (ebd., S. 87).

\section{Historische Epistemologie in der Erziehungswissenschaft}

Forschungen über die Formierung und Transformation erziehungswissenschaftlichen Wissens haben in der Erziehungswissenschaft einen lange Tradition, und seit 1977 treibt eine Arbeitsgemeinschaft, die spätere Kommission für Wissenschaftsforschung in der Deutschen Gesellschaft für Erziehungswissenschaft, „eine ,institutionalisierte‘ Reflexion des Wandels, des Verhältnisses und der Weiterentwicklung erziehungswissenschaftlicher Konzepte und Methoden in wissenschaftstheoretischer, wissenschaftssystematischer und wissenschaftshistorischer Hinsicht“ (Herrmann, zit. nach Horn 2002, S. 182) voran. ${ }^{3}$ Zahlreiche historische Rekonstruktionen befassen sich mit Disziplin- oder Professionsgeschichten (z. B. Glaser \& Andresen 2009; Groppe, Horn \& Kluchert 2010; Hofstetter \& Schneuwly 2011) oder mit theoretischen und methodischen Strömungen in der Erziehungswissenschaft (zuletzt Dinkelaker et al. 2016). Wissenschaftsforschung, Wissenschaftsphilosophie und -geschichte verfolgen dabei nur selten die Perspektive der historischen Epistemologie. Das zeigt sich auch in Wörterbüchern und Lexika, die das Stichwort „Wissen“ aufnehmen (Höhne 2009; Horn et al. 2011). Binder (2014) macht aus systemtheoretischer Perspektive auf die Auslassung einer systematischen Analyse erziehungswissenschaftlicher Wissensproduktion aufmerksam, und Casale (2004) schlägt vor, mit Skinners politischer Ideengeschichte und Foucaults Geschichte der Wissenspraktiken die Disziplingeschichte durch eine Wissensgeschichte zu ergänzen. Eine solche Geschichte müsste die Beziehung zwischen verschiedenen Wissenschaftsgeschichten, das Verhältnis zwischen der Erziehungswissenschaft und der Entwicklung ihrer institutionellen Einbettung sowie die kulturellen Traditionen berücksichtigen, die die Geschichte der Disziplin beeinflusst haben (ebd., S. 404).

\footnotetext{
${ }^{3}$ Vgl. auch die aktuelle Dokumentation auf der Website der Deutschen Gesellschaft für Erziehungswissenschaft (DGfE) http://www.dgfe.de/sektionen-kommissionen/sektion-2-allgemeine-erziehungswissenschaft/kommissionwissenschaftsforschung.html (Zugriff: 14.8.2017).
} 
Explizit auf die historische Epistemologie rekurriert Tenorth (2016), um Erziehungswissenschaft als „wissenschaftliche Praxis eigener Art und eigener Geschichte“ (ebd., S. 33) zu untersuchen. Eine hinreichende Darstellung aus der Perspektive der historischen Epistemologie sei dann gegeben, wenn neben der historischen Betrachtung der Erziehungswissenshaft als institutionalisiertes Wissenssystem auch die epistemologischen Rationalitätslogiken in eine Analyse einbezogen würden.

Die Beiträge zur Geschichte des Begriffs „Aufmerksamkeit“ (Reh et al. 2015) verfolgen diese Intention. Ihnen geht es darum, die Historizität des Phänomens zu rekonstruieren und „Aufmerksamkeit als eine sich transformierende Praktik in ihren institutionellen Bezügen innerhalb des pädagogischen Feldes zu beschreiben und zu analysieren, wie sie in eben diesem Feld eine besondere Funktion als Bestandteil eines Subjektivierungsdispositivs gewinnt“ (ebd., S. 10f.).

Verschiebungen in der erziehungswissenschaftlichen Analyse der Begriffe Wissen, Wissenspraktiken und Wissensgeschichte dokumentieren zwei Beihefte der Zeitschrift für Pädagogik. Oelkers und Tenorth (1991) haben unter dem Titel „Pädagogisches Wissen“ den Anspruch, die Funktionen, Strukturen und Charakteristika der unterschiedlichen Erscheinungsformen von Wissen zu beschreiben und Möglichkeiten der Verbesserung von pädagogischem Wissen aufzuzeigen. 2017 erscheint „Wissen machen. Beiträge zu einer Geschichte erziehungswissenschaftlichen Wissens in Deutschland zwischen 1945 und 1990“, das 63. Beiheft der Zeitschrift für Pädagogik (Reh et al. 2017), mit dem die Herausgeberinnen und der Herausgeber „den Blick auf einen Ansatz lenken, der sich programmatisch mit der historiographischen Rekonstruktion und Analyse der jeweiligen historischen Struktur, Herstellung und Geltung von Wissen [...] befasst“ (Behm et al., 2017, S. 7f.).

Behm (2017) untersucht die Formierung der Bildungsforschung in Westdeutschland, und ihre Hauptthese ist, dass die Wurzeln der Bildungsforschung in der Planungsphase ab 1946 bei der Hochschule für Internationale Pädagogische Forschung liegen. Anhand von Gründungsunterlagen untersucht sie Prozesse, Modi und Aspekte der Wissenskonstruktion und fragt nach zeitlichen (Dis-)Kontinuitäten, die für die Stabilisierung, Auflösung und Transformation von Wissen maßgeblich sind.

Ebenfalls aus einer wissensgeschichtlichen Perspektive analysiert Glaser (2017) den Denkstil und das Denkkollektiv des Deutschen Ausschusses für das Erziehungs- und Bildungswesen in Bezug auf Binnenverhältnisse der Ausschussarbeit, der Zirkulation von Wissen und der Repräsentation nach außen. 
Der Untersuchungsgegenstand von Grube (2017) ist die Bedeutung des Instituts für Demoskopie Allensbach für die Konturierung des westdeutschen bildungspolitischen Wissensfeldes zwischen den 1950er und 1980er Jahren. Er rekonstruiert die Produktion und Zirkulation des Wissens an der Schnittstelle von Gesellschaft, Wissenschaft und Politik. Dabei werden konfligierende Interessen im Denkkollektiv deutlich, aber die Frage bleibt offen, wie die Emergenz widerstreitender Positionen erklärt werden kann und warum sich eine bestimmte Wissensform als hegemonial durchsetzt.

Im Unterschied zu früheren Studien über pädagogisches Wissen rücken nun die Wissensproduzentinnen und -produzenten sowie die Orte und Modi der Wissensproduktion in den Vordergrund. Weitere Forschungen müssten zeigen, welche Rationalitätslogiken für die Formierung und Transformation von Wissen verantwortlich sind und wo Brüche in der Produktion erziehungswissenschaftlichen Wissens zu einer Neuorganisation von Begriffen und Theorien führen.

\section{Ausblick}

Worin besteht die Funktion der historischen Epistemologie für die Erziehungswissenschaft? Man kann die Geschichte einer Disziplin als ihr Unbewusstes begreifen: „das Unbewusste, das sind die verborgen gehaltenen, vergessenen sozialen Bedingungen der Produktion“ (Bourdieu 1993, S. 79). Wenn man begreift, auf welche Weise die Erziehungswissenschaft sich als diejenige Disziplin formiert hat, die sie heute ist, welche Begriffe und Theorien ihre Problematisierung beschreiben, dann ist es auch möglich, „Abwesenheiten“ (Santos 2014, S. 118) zu identifizieren, die in der Geschichte der westlichen Moderne und ihren disziplinären Kategorisierungen aufgehoben sind. Historische Epistemologie kann dazu beitragen, die Erziehungswissenschaft weiterzuentwickeln, indem sie die Geschichte ihrer Wissensproduktion systematisch untersucht. Das schließt zwei wichtige Dimensionen ein: Erstens muss sie mit einem offenen Wissensbegriff operieren und die Interdependenzen zwischen verschiedenen Wissensformen untersuchen, und zweitens darf sie dabei nicht von einem vorgängigen Begriff der Disziplin und ihrer Grundbegriffe ausgehen. Historische Epistemologie ist kein normatives Unterfangen, sie macht vielmehr normative Dimensionen der Wissensproduktion zu ihrem Gegenstand. Wenn die Forschungspraxis stärker in den Fokus der Wissensproduktion rückt, dann eröffnen sich bisher noch zu wenig genützte Anschlussmöglichkeiten an Science and Technology Studies und, theoretisch und methodisch, an Netzwerkanalysen, um unterschiedliche Denkkollektive und die Machtverhältnisse von Wissenspraktiken präziser bestimmen zu können (Glaser 2017, S. 103). 
Schließlich weisen wir mit Rheinberger (2017, S. 43) darauf hin, dass Forschungen zur historischen Epistemologie selbst einer Geschichte unterliegen. Eine der wichtigen Herausforderungen liegt in einer reflexiven Historisierung der historischen Epistemologie selbst, das heißt, in einer doppelt reflexiven Historisierung der Erziehungswissenschaft.

\section{Literatur}

Bachelard, G. (1980). Die Philosophie des Nein. Versuch einer Philosophie des neuen wissenschaftlichen Geistes (1940). Frankfurt a.M.: Suhrkamp.

Bachelard, G. (1987). Die Bildung des wissenschaftlichen Geistes. Beitrag zu einer Psychoanalyse der objektiven Erkenntnis (1938). Frankfurt a.M.: Suhrkamp.

Barad, K. (2015). Verschränkungen. Berlin: Merve.

Bauer, S., Heinemann, T., \& Lemke, T. (2017). Science and Technology Studies. Klassische Positionen und aktuelle Perspektiven. Frankfurt a.M.: Suhrkamp.

Behm, B. (2017). Zu den Anfängen der Bildungsforschung in Westdeutschland 1946-1963. Ein wissensgeschichtlicher Blick auf eine ,vergessene' Geschichte. In: S. Reh, E. Glaser, B. Behm, \& T. Drope (Hrsg.), Wissen machen. Beiträge zu einer Geschichte erziehungswissenschaftlichen Wissens in Deutschland zwischen 1945 und 1990 (63. Beiheft der Zeitschrift für Pädagogik) (S. 34-69). Weinheim: Beltz.

Behm, B., Drope, T., Glaser, E., \& Reh, S. (2017). Wissen machen - Beiträge zu einer Geschichte erziehungswissenschaftlichen Wissens in Deutschland zwischen 1945 und 1990. Einleitung zum Beiheft. In: S. Reh, E. Glaser, B. Behm, \& T. Drope (Hrsg.), Wissen machen. Beiträge zu einer Geschichte erziehungswissenschaftlichen Wissens in Deutschland zwischen 1945 und 1990 (63. Beiheft der Zeitschrift für Pädagogik) (S. 7-15). Weinheim: Beltz.

Binder, U. (2014). Wie erziehungswissenschaftliches Wissen erzeugt wird. Eine Strukturanalyse. Vierteljahrsschrift für wissenschaftliche Pädagogik 4, 591-611.

Borck, C., Hess, V., \& Schmidgen, H. (2005). Erkenntnis des Lebenden. Eine Skizze zu Georges Canguilhem (1904-1995). Berlin: Max-Planck-Institut für Wissenschaftsforschung.

Bourdieu, P. (1993). Soziologische Fragen. Frankfurt a.M.: Suhrkamp.

Canguilhem, G. (1974). Das Normale und das Pathologische. München: Hanser.

Canguilhem, G. (1979). Wissenschaftsgeschichte und Epistemologie. Gesammelte Aufsätze. Frankfurt a.M.: Suhrkamp.

Casale, R. (2004). The educational theorists, the teachers, and their history of education. A plea for a history of educational knowledge. Studies in Philosophy and Education 23, 393-408.

Daston, L., \& Galison, P. (2007). Objektivität. Frankfurt a.M.: Suhrkamp.

Daston, L. (2009). Science studies and the history of science. Critical Inquiry 35(4), 798-813.

Dear, P., \& Jasanoff, S. (2010). Dismantling boundaries in science and technology studies. Isis 101, 759-774.

Deleuze, G. (1987). Foucault. Frankfurt a.M.: Suhrkamp.

Dinkelaker, J., Meseth, W., Neumann, S., \& Rabenstein, K. (2016). Die Erziehungswissenschaft, ihr Gegenstand und ihre Empirie. Sondierungen im Spannungsfeld von traditionellen Kontroversen und reflexiver Empirisierung. In: W. Meseth, J. Dinkelaker, S. Neumann, K. Rabenstein, O. Dörner, M. Hummrich, \& K. Kunze (Hrsg.), Empirie des Pädagogischen und Empirie der Erziehungswissenschaft (S. 13-30). Bad Heilbrunn: Klinkhardt.

Fleck, L. (1980). Entstehung und Entwicklung einer wissenschaftlichen Tatsache. Einführung in die Lehre von Denkstil und Denkkollektiv (1935). Frankfurt a.M.: Suhrkamp. 
Fleck, L. (2011). Das Problem einer Theorie des Erkennens (1936). In: Ders., Denkstile und Tatsachen. Gesammelte Schriften und Zeugnisse (S. 260-309). Berlin: Suhrkamp.

Foucault, M. (1981). Archäologie des Wissens. Frankfurt a.M.: Suhrkamp.

Foucault, M. (2003/1978). Vorwort von Michel Foucault. In : M. Foucault, Dits et Ecrits. Schriften. Dritter Band (S. 551-567). Frankfurt a.M.: Suhrkamp.

Gingras, Y. (2010). Naming without necessity. On the genealogy and uses of the label ,historical epistemology'. Revue de synthèse 131(3), 439-454.

Glaser, E., \& Andresen, S. (Hrsg.) (2009). Disziplingeschichte der Erziehungswissenschaft als Geschlechtergeschichte. Jahrbuch Frauen- und Geschlechtergeschichte in der Erziehungswissenschaft, Bd. 5.

Glaser, E. (2017). Pädagogik und Politik. Der Deutsche Ausschuss für das Erziehungs- und Bildungswesen und seine Empfehlungen als ein Beitrag zur Wissensgeschichte in der frühen Bundesrepublik. In: S. Reh, E. Glaser, B. Behm, \& T. Drope (Hrsg.), Wissen machen. Beiträge zu einer Geschichte erziehungswissenschaftlichen Wissens in Deutschland zwischen 1945 und 1990 (63. Beiheft der Zeitschrift für Pädagogik) (S. 88-107). Weinheim: Beltz.

Groppe, C., Horn, K.-P. \& Kluchert, G. (Red.) (2010). Jahrbuch für historische Bildungsforschung 2009 (Bd. 15). Schwerpunkt Wissenschaftsgeschichte. Bad Heilbrunn: Klinkhardt.

Grube, N. (2017). Bildungspolitische Beratung durch das Allensbacher Institut für Demoskopie in der Bundesrepublik von 1950 bis zum Ende der 1980er Jahre. In: S. Reh, E. Glaser, B. Behm, \& T. Drope (Hrsg.), Wissen machen. Beiträge zu einer Geschichte erziehungswissenschaftlichen Wissens in Deutschland zwischen 1945 und 1990 (63. Beiheft der Zeitschrift für Pädagogik) (S. 70-87). Weinheim: Beltz.

Hacking, I. (1999). Was heißt ,soziale Konstruktion‘? Zur Konjunktur einer Kampfvokabel in den Wissenschaften. Frankfurt a.M.: Fischer.

Haraway, D. (1995). Die Neuerfindung des Sozialen. Primaten, Cyborgs und Frauen. Frankfurt a.M.: Campus.

Hofstetter, R., \& Schneuwly, B. (2011). Zur Geschichte der Erziehungswissenschaften in der Schweiz. Vom Ende des 19. bis zur Mitte des 20. Jahrhunderts. Bern: hep.

Höhne, T. (2009). Wissen. In: S. Andresen, R. Casale, T. Gabriel, R. Horlacher, S. Larcher Klee, \& J. Oelkers (Hrsg.), Handwörterbuch Erziehungswissenschaft (S. 897-911). Weinheim: Beltz.

Horn, K.-P. (2002). Zur Geschichte der Arbeitsgemeinschaft für Wissenschaftsforschung (AfW) bzw. Kommission AG Wissenschaftsforschung der Deutschen Gesellschaft für Erziehungswissenschaft. Tagungen, Publikationen, Themen. Zeitschrift für Erziehungswissenschaft 5(Beiheft 1), 181-211.

Horn, K.-P., Kemnitz, H., Marotzki, W., \& Sandfuchs, U. (Hrsg.) (2011). Klinkhardt Lexikon Erziehungswissenschaft (KLE). Bad Heilbrunn: Klinkhardt.

Kuhn, T. S. (1973). Die Struktur wissenschaftlicher Revolutionen. Frankfurt a.M.: Suhrkamp.

Latour, B. (1995). Wir sind nie modern gewesen. Versuch einer symmetrischen Anthropologie. Berlin: Akademie.

Lecourt, D. (1969). L'épistémologie historique de Gaston Bachelard. Paris: Vrin.

Limoges, C. (2012). L'épistémologie historique dans l'itinéraire intellectuel de Georges Canguilhem. In: H. Schmidgen, P. Schöttler, \& J.-F. Braunstein (Hrsg.), Epistemology and history. From Bachelard and Canguilhem to today's history of science (S. 53-66). Berlin: Max-Planck-Institut für Wissenschaftsgeschichte.

Messerschmidt, A. (2012). Michel Foucault (1926-1984). Den Befreiungen misstrauen Foucaults Rekonstruktionen moderner Macht und der Aufstieg kontrollierter Subjekte. In: B. Dollinger (Hrsg.), Klassiker der Pädagogik. Die Bildung der modernen Gesellschaft (S. 289-310). Wiesbaden: VS Verlag. 
Müller, E., \& Schmieder, F. (2016). Begriffsgeschichte und historische Semantik. Ein kritisches Kompendium. Frankfurt a.M.: Suhrkamp.

Oelkers, J., \& Tenorth, H.-E. (Hrsg.) (1991). Pädagogisches Wissen (27. Beiheft der Zeitschrift für Pädagogik). Weinheim: Beltz.

Reh, S., Berdelmann, K., \& Dinkelaker, J. (Hrsg.) (2015). Aufmerksamkeit. Geschichte - Theorie - Empirie. Wiesbaden: Springer.

Reh, S., Glaser, E., Behm, B., \& Drope, T. (Hrsg.) (2017). Wissen machen - Beiträge zu einer Geschichte erziehungswissenschaftlichen Wissens in Deutschland zwischen 1945 und 1990 (63. Beiheft der Zeitschrift für Pädagogik). Weinheim: Beltz.

Renn, J. (Hrsg.) (2012). The globalization of knowledge in history. Berlin: Max-PlanckInstitut für Wissenschaftsgeschichte.

Rheinberger, H.-J. (2006). Epistemologie des Konkreten. Studien zur Geschichte des Konkreten. Studien zur Geschichte der modernen Biologie. Frankfurt a.M.: Suhrkamp.

Rheinberger, H.-J. (2007). Historische Epistemologie. Eine Einführung. Hamburg: Junius.

Rheinberger, H.-J. (2017). Historische Epistemologie. In: M. Sommer, S. Müller-Wille, \& C. Reinhardt (Hrsg.), Handbuch Wissenschaftsgeschichte (S. 32-45). Stuttgart: Metzler.

Ricken, N., \& Rieger-Ladich, M. (Hrsg.) (2004). Michel Foucault: Pädagogische Lektüren. Wiesbaden: VS Verlag.

Roelcke, V. (2010). Auf der Suche nach der Politik in der Wissensproduktion: Plädoyer für eine historisch-politische Epistemologie. Berichte zur Wissenschaftsgeschichte 33, 176-192.

Santos, B. d. S. (2014). Epistemologies of the south. Justice against epistemicide. Boulder: Paradigm.

Sarasin, P. (2011). Was ist Wissensgeschichte? Internationales Archiv für Sozialgeschichte der deutschen Literatur 36(1), 159-172.

Schmidgen, H., Schöttler, P., \& Braunstein, J.-F. (Hrsg.) (2012). Epistemology and history. From Bachelard and Canguilhem to today's history of science. Berlin: Max-PlanckInstitut für Wissenschaftsgeschichte.

Sommer, M., Müller-Wille, S., \& Reinhardt, C. (Hrsg.) (2017). Handbuch Wissenschaftsgeschichte. Stuttgart: Metzler.

Speich Chassé, D., \& Gugerli, D. (2012). Wissensgeschichte. Eine Standortbestimmung. Traverse. Zeitschrift für Geschichte 19(1), 85-100.

Tenorth, H.-E. (2016). „Erziehungswissenschaft“ - In: W. Meseth, J. Dinkelaker, S. Neumann, K. Rabenstein, O. Körner, M. Hummrich, \& K. Kunze (Hrsg.), Empirie des Pädagogischen und Empirie der Erziehungswissenschaft. Beobachtungen erziehungswissenschaftlicher Forschung (S. 33-54). Bad Heilbrunn: Klinkhardt.

Wulz, M. (2014). Technik im Wissen: Zur wechselseitigen Hervorbringung von Wissen, Technik, Geschichte und Gesellschaft in der französischen Wissenschaftsgeschichte und -philosophie. In: D. Lengersdorf, \& M. Wieser (Hrsg.), Schlüsselwerke der Science \& Technology Studies (S. 67-83). Wiesbaden: Springer. 